\title{
Sobre as especies do genero Capillaria Zeder, 1800, parasitas de morcegos (*)
}

\author{
por
}

\section{J. F. TEIXEIRA DE FREITAS}

(Com as estampas XLI-XLIII)

Encontramos na literatura 4 especies do genero Capillaria, parasitas de Chiropteros. Estudando material incluido na coleção do Instituto Oswaldo Cruz tivemos ocasião de encontrar algumas especies que consideramos novas. Fizemos, então, um estudo de conjunto das especies, que serão descritas na presente publicação, segundo a ordem cronologica de suas descrições.

\section{1) Capillaria vespertilionis (Rudolphi, 1819)}

Sin.: - Trichosoma vespertilionis Rudolphi, 1819, p. 14.

Trichosomum vespertilionis Diesing, 1851, p. 258.

Trichosomum vespertilionis Kolenati, 1856, pp. 13-14.

Trichosomum vespertilionis Kolenati, 1857, idem.

Capillaria vespertilionis Travassos, 1915, p. 159.

Capillaria vespertilionis Yorke \& Maplestone, 1926, p. 27.

Trichosoma vespertilionis Stiles \& Nolan, 1931, n. ${ }^{\circ} 155$, p. 623.

Esta especie referida por Rudolphi entre as especies duvidosas, é encontrada no intestino de Nyctalus noctula (Schreb.). É esta, aliás, a unica indicação fornecida por este autor, retirada do Catalogo do Museu de Viena. Dujardin, á pag. 9 da sua Hist. Nat. de Helm. (1845), diz ter sido encontrada nesse hospedador uma especie indeterminada, deste genero. Diesing e Kolenati tambem a ela fazem referencia, citando mais um hospedador - Vespertilio discolor (Natterer). Travassos a considera nom. nud.

Julgamos acertado continuar esta especie como duvidosa, até uma ulterior determinação feita em material colhido no hospedador a que se refere Rudolphi, isto é, no Nyctalus noctula (Schreb.).

(*) Recebido para publicação a 18 de Março de 1934. 
Habitat: Intestino delgado de $N$. noctula (Schreb.) e Vespertilio discolor (Natt.).

\section{2) Capillaria diesingii (Kolenati, 1856)}

SIn. : - Trichosomum diesingii Kolenati, 1856, p. 13.

Trichosomum diesingii Kolenati, 1857, idem.

Capillaria diesingii Stiles \& Nolan, 1931, n. ${ }^{\circ}$ 155, p. 623.

Désta especie o autor não encontrou exemplares femeas, e para o macho dá de comprimento $15 \mathrm{~m} m$. e de largura $0,12 \mathrm{~mm}$. Descreve ainda os seguintes caracteres: cabeça dilatada em fórma de botão com uma papila terminal; iextremidade posterior dos machos provida de 3 ganchos recurvados para o penis.

Haвitat: - Intestino delgado de Myotis murinus (Schreb.).

3) Capillaria speciosa (v. Beneden, 1873).

SIN.: - Trichosomum speciosum v. Beneden, 1873, pp. 19-20, pl. 4, 6 figs. Trichosomum speciosum v. Beneden, 1873, pp. 319-321.

Trichosomum speciosum Linstow, 1878, p. 13.

Trichosomum speciosum Cobbold, 1879, p. 294.

Trichosoma speciosum Stossich, 1890, p. 8.

Trichosoma speciosum Linstow, 1909, p. 80, fig. 77.

Capillaria speciosa Travassos, 1915, p. 151, 152.

Capillaria speciosa Yorke \& Maplestone, 1926, p. 27.

Capillaria speciosa Stiles \& Nolan, 1931, n. ${ }^{\circ}$ 155, p. 623.

Comprimento: - macho $13 \mathrm{~mm}$; femea $20 \mathrm{~mm}$.

Largura: - 0,2 a $0,4 \mathrm{~mm}$.

Boca desguarnecida. Corpo da femea atenuado nas 2 extremidades. Femiea com vulva situada no meio do comprimento do corpo, abrindo-se na extremidade de uma saliencia alongada semelhante a um penis. Utero mais ou menos repleto de ovos, bi-operculados, de casca resistente, que possuem $0,25 \mathrm{~mm}$. de comprimento. Extremidade posterior mais ou menos afilada. Macho provido de espiculo grande, medindo $1 / 4$ do comprimento do corpo. $(3,25 \mathrm{~mm}$.). Bainha espicular lisa e fortemente estriada no sentido transversal. Orificio cloacal sub-terminal. Extremidade caudal apresenta duas azas membranosas, uma de cada lado, sustentadas por meio de raios pouco numerosos e fracamente acusados. Nesta especie a relação entre a porção anterior e a posterior é igual a $28: 27$. 
Junho, 1934

T. de Freitas: Capillaria parasitas de morcegos

Habitat: Estomago de:-Myotis dasycneume (Boie.); Myotis nattereri (Kuhl.); Myotis daubentonii (Leisler); Eptesicus serotinus (Schreb.);

Distribuição GeOgrafica: Belgica. e Vespertilio murinus (Natt.).

4) Capillaria pusilla Travassos, 1914

(Estampa XLI, figs. 1-2).

Sin. : - Capillaria pusilla Travassos, 1914, p. 429.

Capillaria pusilla Travassos, 1915, p. 153, est. 24, fig. 7.

Capillaria pusilla Yorke \& Maplestone, 1926, p. 27, 28.

Capillaria pusilla Stiles \& Nolan, 1931, n. ${ }^{\circ}$ 155, p. 623:

Redescrição: Comprimento:- macho 9,1 a $9,5 \mathrm{~mm}$.; femea $14,7 \mathrm{~mm}$.

Largura: - macho 0,042 a $0,056 \mathrm{~mm}$; femea 0,039 a $0,100 \mathrm{~mm}$.

Corpo de côr branca, com estriações transversais muito finas, atenuado anteriormente; bôca desguarnecida; esofago muito longo mede cerca de 3 a $5 \mathrm{~mm}$.

Femea com vulva situada mais ou menos a $3,2 \mathrm{~mm}$. da extremidade anterior (est. XLI, fig. 1), com labios salientes, sobretudo o anterior, que fórma mesmo uma projeção bastante nitida; o labio posterior possue 2 saliencias ou papilas arredondadas. Vagina longa de 0,13 a $0,14 \mathrm{~mm}$; utero cheio de ovos de 0,056 a $0,063 \mathrm{~mm}$. de comprimento por 0,024 a $0,031 \mathrm{~mm}$. de maior largura. Orificio anal quasi terminal (est. XLI, fig. 2). Relação entre a porção anterior e a posterior é igual a $1: 3$.

Macho com espiculo de $0,56 \mathrm{~mm}$. de comprimento por $0,005 \mathrm{~mm}$, de largura, acompanhado de bainha espessa, com estriação transversal muito nitida, sinuosa e sem espinhos. Extremidade caudal com 2 papilas situadas lateralmente. Cloaca terminal. Relação entre a porção anterior e a posterior é de $1: 3$.

Habitat: Intestino delgado de Sturnira lilium Geoff.

Distribuição geografica: Manguinhos, Rio de Janeiro, Brasil.

Esta redescrição é baseada nos tipos encontrados na coleção do Instituto Osivaldo Cruz.

\section{5) Capillaria pintoi n. sp.}

(Estampa XLI, figs. 3-5; Estampa XLII, fig. 6),

Comprimento:-macho $4,7 \mathrm{~mm}$.; femea $10,1 \mathrm{~mm}$.

Largura: - macho $0,056 \mathrm{~mm}$; femea $0,157 \mathrm{~mm}$. 
Corpo atenuado anteriormente, com bôca sem guarnições; esofago com $2,5 \mathrm{~mm}$. a $3,8 \mathrm{~m} / \mathrm{m}$. de comprimento.

Fiemea com extremidade posterior obtusa, de anus sub-terminal (est. XLI, fig. 4). A vulva apresenta no labio anterior um apendice volumoso constituido de uma parte basal forte e estriada transversalmente e de outra parte terminal, membranosa, muito fina e pregueada longitudinalmente (est. XLI, fig. 3). O comprimento desse apendice vulvar é de $0,104 \mathrm{~mm}$. ıe sua largura é de 0,056 a $0,064 \mathrm{~mm}$. Utero com ovos de 0,051 a $0,054 \mathrm{~mm}$. de comprimento por 0,032 a $0,035 \mathrm{~mm}$. de largura. Relação entre a porção anterior e a posterior é de $1: 8$.

Macho com espiculo longo de 0,57 mm., apresentando uma estriação transversa muito nitida (est. XLI, fig. 5). Bainha espicular sem espinhos. Extremidade caudal com 2 processos dorsais e 2 ventrais reunidos entre si formando um conjunto fracamente quitinisado com uma concavidade dirigida posteriormente (est. XLII, fig. 6). Orificio cloacal terminal. Relação entre a porção anterior e a posterior é de $1: 2$.

Habitat: Intestino delgado de Chiroptera.

Distribuição geografica: Lassance, Estado de Minas Gerais, Brasil.

Dedicamos esta especie, da qual examinamos um macho e uma femea, ao Dr. Cesar Pinto, que fez a colheita do material.

Tipos no Instituto Oswaldo Cruz.

\section{6) Capillaria pulchra n. sp.}

(Estampa XLII, fig. 7; Estampa XLIII, figs. 11-12).

Comprimento: - femea $15,6 \mathrm{~mm}$.

Largura: - macho $0,12 \mathrm{~mm}$; femea $0,18 \mathrm{~mm}$.

Corpo de cuticula estriada transversalmente; bôca circular e núa; esofago longo de $5,4 \mathrm{~mm}$.

Femea com vulva provida de uma saliencia globosa, tendo de comprimento $0,056 \mathrm{~mm}$. e de largura 0,040 a $0,048 \mathrm{~mm}$. (est. XLIII, fig. 11). Utero cheio de ovos de $0,048 \mathrm{~mm}$. de comprimento por 0,032 $\mathrm{mm}$. de largura. Extremidade posterior um pouco curvada no sentido ventral; apresentando o anus situado sub-terminalmente (est. XLIII, fig. 12). Relação entre a porção anterior e a posterior é de $1: 3$.

Macho com espiculo de $0,14 \mathrm{~mm}$. de comprimento, acompanhado de bainha espinosa. Extremidade posterior apresenta duas azas caudais bem desenvolvidas e 4 papilas, sendo 2 ventrais e volumosas, inteiramente separadas desde a sua base, e outras 2 dorsais, pequenas e finas, 
nascendo de uma base comum bastante larga (est. XLII, fig. 7). Orificio cloacal sub-terminal.

Habitat: Estomago de Nyctinomus brasiliensis Geoff.

Distribuição geografica: Manguinhos, Rio de Janeiro, Brasil.

Tipos no Instituto Oswaldo Cruz. gmentados.

Desta especie examinamos 2 femeas inteiras e 2 machos fra-

\section{7) Capillaria angrense n. sp.}

(Estampa XLII, figs. 8-9; Estampa XLIII, fig. 10).

Comprimento: - macho 13,4 a $13,5 \mathrm{~mm}$.; femea 17,7 a $18,0 \mathrm{~mm}$. Largura: - macho $0,07 \mathrm{~m} / \mathrm{m}$; femea 0,05 a $0,10 \mathrm{~mm}$.

Corpo atienuado anteriormente, com cuticula apresentando finas estrias transversas bem visiveis na extremidade posterior; bôca circular e desprovida de papilas; esofago de celulas muito nitidas, mede de 5,4 a $6,8 \mathrm{~mm}$., sendo $0,37 \mathrm{~mm}$. para a sua porção muscular.

Fiemea com vulva situada a $0,104 \mathrm{~mm}$. da terminação do esofago, de labios salientes, possuindo o anterior uma membrana cuticular de $0,064 \mathrm{~mm}$. de comprimento por $0,048 \mathrm{~mm}$. de largura (est. XLIII, fig. 10). Vagina curta, á qual se segue um utero com ovos de 0,037 a $0,043 \mathrm{~mm}$. de comprimento por $0,024 \mathrm{~mm}$. de largura. Em algumas das femeas encontramos os ovos já apresentando uma larva no interior. Extremidade posterior obtusa, porém muito menos larga que o restoi do corpo (0,024 mm. de largura) (est. XLII, fig. 8). Orificio anal subterminal. Relação entre a porção anterior e a posterior é de $1: 3$.

Macho com espiculo de 0,37 a $0,41 \mathrm{~mm}$. de comprimento. Bainha espicular apresenta 2 porções bem distintas: uma terminal, lisa, que nos 2 exemplares examinados se apresentava desenvaginada, semelhando um prolongamento da parte externa do corpo do nematodeo, e outra; mais interna, recoberta por espinhos pequenos (est. XLII, fig. 9). A porção não espinhosa da bainha, que se apresentava sempre com algumas pregas, mede de comprimento 0,09 a $0,13 \mathrm{~mm}$. e de largura média 0,054 a $0,056 \mathrm{~mm}$. Extremidade caudal mostra uma papila fina, de posição dorsal, e 4 pares de papilas dirigidas ventralmente, situadas 2 pares de cada lado. Notam-se 2 azas caudais bem desenvolvidas, pre-cloacais, e 2 expansões cuticulares post-cloacais, que formam o extremo posterior do corpo do helminto. Cloaca subterminal. Ambos os machos examinados apresentavam a extremidade caudal um pouco curvada para a parte dorsal. Relação entre a porção anterior e a posterior é de $2: 5$. 
Habitat: Intestino de Molossus rufus Geoff.

Distribuição geografica: Angra dos Reis, Estado do Rio, Brasil.

Desta especie examinamos 2 casais perfeitos e algumas femeas fragmentadas.

Tipos no Instituto Oswaldo Cruz.

\section{BIBLIOGRAFIA}

VAN BENEDEN, 1873 - Les parasites des chauves-souris de Belgique, Mém. de la Acad. R. des Sc., t. XL, pp. 3-24, pl. I-VII.

* VAN BENEDEN, 1873 - Vers parasites des chauves-souris de la Belgique, Jl. de Zoologie, vol. 2, pp. 319-321.

COBBOLD, 1879 - Parasites, a treatise on the Entozoa of man and animals including some account of the ectozoa, London.

DIESING, 1851 - Systema Helminthum, II.

DUJARDIN, 1845 - Histoire naturelle des Helminthes, Paris.

* KOLENATI, 1856 - Die Parasiten der Chiroptern.

KOLENATI, 1857-Idem, idem, Dresden.

V. LINSTOW, 1878 - Compendium der Helminthologie, Hannover.

V. LINSTOW, 1909 - Parasitische Nematoden. Die Süsswasserfauna Deutschlands, pp. 47-83, 80 figs.

RUDOLPHI, 1819 - Entozoorum synopsis, Berolini.

STILES, C. W. \& NOLAN, M. O., 1931-Key Catalogue of Parasites reported for Chiroptera (bats) with their possible public health importance. National Inst. of Health. Bull., n. ${ }^{\circ} 155$.

STOSSICH, M., 1890 - Il genere Trichosoma Rudolphi, Trieste.

TRAVASSOS, L., 1914 - Sobre as especies brasileiras do genero " $\mathrm{Ca}$ pillaria Zeder, 1800 ", Brasil-Medico, ano 28, n. ${ }^{\circ}$ 47, p. 429.

TRAVASSOS, L., 1915 - Contribuições para o conhecimento da fauna helmintologica brasileira. V. - Sobre as especies brasileiras do genero Capillaria Zeder, 1800, Mem. Inst. O. Cruz, 1. VII, f. II, pp. 146-171, est. 2326, 14 figs.

YORKE, W. \& MAPLESTONE, P. A.. 1926- The Nematode parasites of Vertebrates, London.

(*) Os trabalhos marcados por este sinal não foram consultados. 


\section{EXPLICAÇÃO DAS ESTAMPAS XLI-XLIII}

\section{ESTAMPA XLI}

Fig. 1-Capillaria pusilla Travassos, 1914: Região vulvar. Original.

Fig. 2-Capillaria pusilla Travassos, 1914: Extremidade posterior da femea. Original.

Fig. $\quad 3$-Capillaria pintoi n. sp.: Região vulvar.

Fig. 4-Capillaria pintoi n. sp.: Extremidade posterior da femea.

Fig. $\quad 5$-Capillaria pintoi n. sp.: Extremidade terminal do espiculo.

\section{ESTAMPA XLII}

Fig. 6 -Capillaria pintoi n. sp.: Extremidade posterior do macho.

Fig. 7-Capillaria pulchra n. sp.: Extremidade posterior do macho.

Fig. 8-Capillaria angrense n. sp.: Extremidade posterior da femea.

Fig. 9 -Capillaria angrense n. sp.: Extremidade posterior do macho.

\section{ESTAMPA XLIII}

Fig. 10-Capillaria angrense n. sp.: Região vulvar.

Fig 11 -Capillaria pulchra n. sp.: Região vulvar.

Fig. 12-Capillaria pulchra n. sp.: Extremidade posterior da fenea.

(Laboratorio de Helmintologia - Prof. Lauro Travassos). 

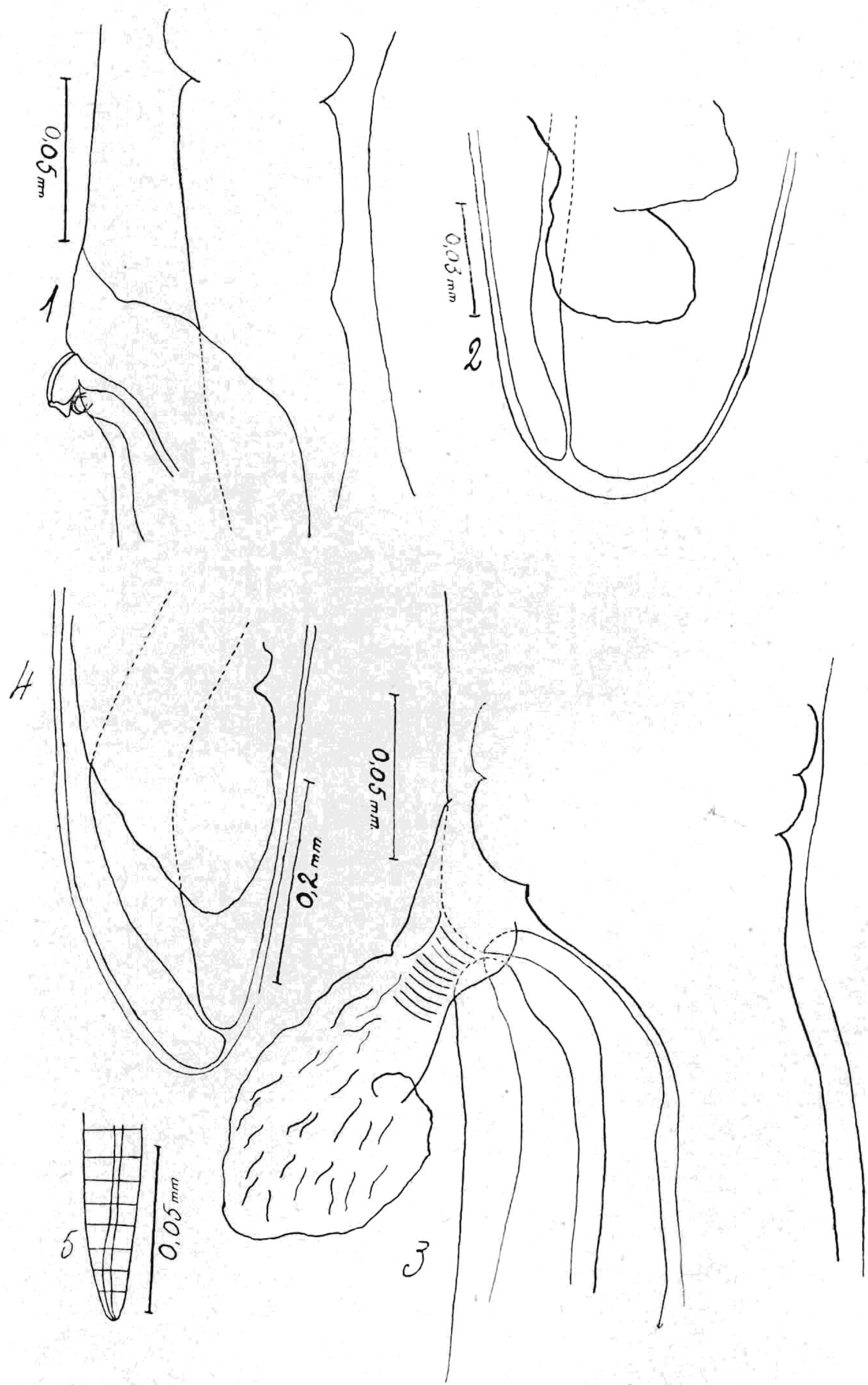

J. F. Teixeira de Freitas : Sobre as especies do genero Capillaria Zeder, 1800, parasitas de morcegos. 

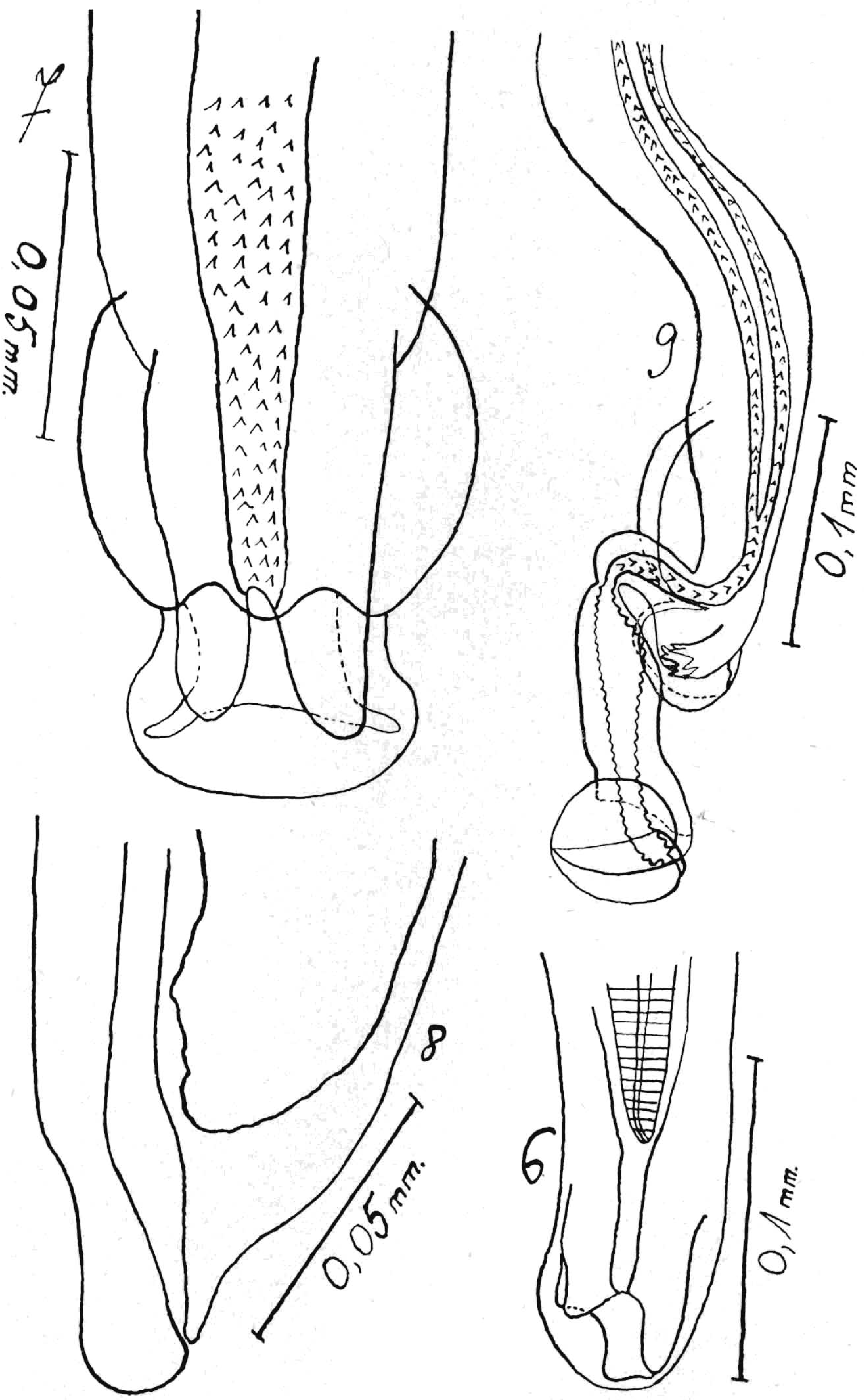

J. F. Teixeira de Freitas : Sobre as especies do genero Capillaria Zeder, 1800, parasitas de morcegos. 


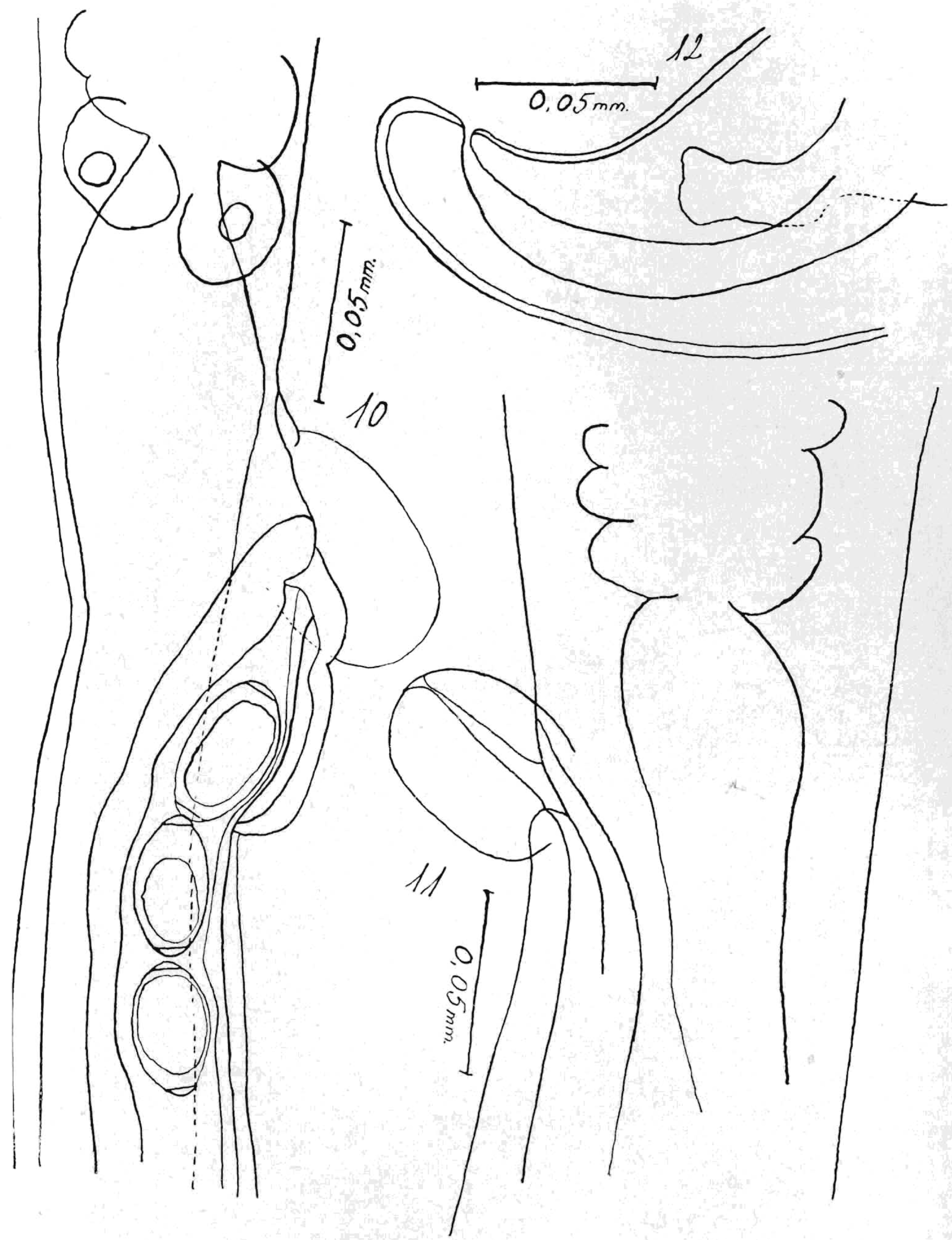

J. F. Teixeira de Freitas : Sobre as especies do genero Capillaria Zeder, 1800, parasitas de morcegos. 